\title{
Transitional care clinics: An innovative approach to reducing readmissions, optimizing outcomes and improving the patient's experience of discharge care-conducting a feasibility study
}

\author{
Melanie Smith* \\ Houston Methodist Hospital, Houston, United States
}

Received: July 14, 2015

DOI: $10.5430 /$ jnep.v6n6p34
Accepted: October 12, $2015 \quad$ Online Published: January 28, 2016

URL: http://dx.doi.org/10.5430/jnep.v6n6p34

\begin{abstract}
Rehospitalization, which is defined as a return to the hospital for the same or related care within 30 days, is often avoidable and adds significant costs to health care spending. A growing body of evidence indicates that when patients move from one health care setting to another, a period known as the care transition, timely access to follow-up care is one of the keys to avoiding unplanned readmissions. Of late, transitional care clinics (TCCs), sometimes referred to as post-discharge clinics, have emerged as an innovative approach to readmission reduction. TCCs bridge the care gap between hospital discharge and post-acute care follow-up by providing access to care for patients with fragmented care or at high risk for readmission. These clinics are typically hospital-based and nurse practitioner-led which makes sense in terms of both cost-containment and quality of care. They provide an alternative to the use of emergency services, improve workflow for referring physicians, and support care navigation back to community providers. TCCs support the discharge plan of care and long-term outcomes by providing an intense focus on patient education, disease and medication self-management, and coordination of care. By providing access to care and improving communication across the continuum of care, TCCs can improve the quality of the care transition and reduce avoidable readmissions. A feasibility analysis can help to provide answers and guide project planning. This article describes how to conduct such a feasibility analysis of establishing a TCC as well as the organizational, financial and market factors impacting the feasibility of establishing such a clinic.
\end{abstract}

Key Words: Transitional care, Hospital discharge, Readmission reduction, Feasibility study, Innovation, Nurse practitioner, Business plan

\section{OVERVIEW}

Health care costs represent approximately one-sixth of the U.S. economy, and this percentage continues to rise. Although home health care spending is increasing at the fastest pace, hospital services account for the greatest portion of both Medicare and personal health expenditures. The strain on health care resources and financing is predicted to continue as the prevalence of chronic illness increases among younger and adult Americans and the growing baby boomer population creates 10,000 entrants into the Medicare program daily. Nearly one-third of the two trillion dollars spent on health care in 2005 paid for hospital-related services: in-

*Correspondence: Melanie Smith; Email: MESmith@houstonmethodist.org; Address: Houston Methodist Hospital, Houston, United States. 
patient care and medications, care received in hospital-based skilled nursing facilities, and post-discharge home-health care services. ${ }^{[1,2]}$ Whereas hospital-based care is often necessary and in most cases appropriate, rehospitalization, which is defined as a return to the hospital for the same or related care within 30 days, is often avoidable and adds significant and unnecessary costs to health care spending. ${ }^{[3]}$ Rehospitalizations occur at an alarming rate. It has been reported that 1 in 5 Medicare beneficiaries who leave the hospital are readmitted within 30 days, and most of those readmissions are unplanned. Avoidable readmissions cost Medicare more than $\$ 17$ billion dollars per year and represent a significant source of waste and inefficiency in the current health care delivery system. ${ }^{[3]}$

Unplanned rehospitalizations reflect the quality of care a hospital provided for a patient at discharge. A growing body of evidence indicates that when patients move from one care setting to another, a period referred to as the care transition, continuity of care is one of the keys to avoiding unplanned readmissions. However, continuity is challenging in a complex, poorly connected health care system, particularly when patients are older, have multiple providers, have multiple chronic health problems, or require complex medication management. Discharge handoff information is often poorly communicated and low health literacy or subclinical cognitive derangements frequently go unrecognized; this increases the risk for medication errors, noncompliance, and other adverse events. In addition, many patients lack access to a post-hospital provider when one is most needed. For example, a recent study on hospital readmissions found that less than half of rehospitalized Medicare patients were able to arrange follow-up with a primary care provider within the critical 14-day period following discharge. ${ }^{[4,5]}$ These problems can result in preventable complications that increase the likelihood of readmissions and increase health care system costs. ${ }^{[6]}$

With financial penalties now tied to avoidable readmissions, hospitals have become increasingly focused on improving the quality of transitional care. Some are using comprehensive care coordination strategies that provide a more holistic approach to disease management; others use methods like follow-up phone calls to support patients during the immediate post-discharge period. ${ }^{[7]}$ Of late, however, a new approach to readmission reduction has begun to emerge in hospitals and health systems. For patients with urgent postdischarge needs who lack access to home care services or timely primary care follow-up, a care delivery setting known as the transitional care clinic (TCC) has arrived. ${ }^{[5,6,8]}$

TCCs provide a bridge between hospital discharge and pri-

Published by Sciedu Press mary or specialty care follow-up. They give patients at risk for readmission access to medical care and support for any social or educational needs that develop during the immediate post-discharge period. ${ }^{[4]}$ They are not meant to replace or provide primary care; on the contrary, these clinics are being uniquely designed to limit care delivery to the 30 -day postdischarge period. Because TCCs are typically based at the hospitals patients are discharged from, there is ease of access to hospital records and inpatient providers, and transitional care can be provided in continuity with the hospital discharge plan. TCCs are also a better setting for patient teaching and the handoff of care to the patient or family and community providers. TCCs are designed to provide patients in transition with an alternative to the use of emergency services and to be an active partner with community-based health care providers. By providing access to care and improving communication across the continuum of care, TCCs improve the quality of the care transition. ${ }^{[4-6]}$

Whereas it is easy to see the merit in this innovative concept, evidence is lacking on fiscal or disease management outcomes. Also, there is minimal evidence available showing that hospital readmissions are being impacted. Nevertheless, TCCs are on the rise. Because the needs and resources of every health system and community are unique, the risks and benefits of establishing such a clinic must be evaluated on an individual basis. It is important to predict resource needs and assess market conditions before moving forward with such a huge collaborative undertaking. In addition, the financial impact a TCC is likely to have on a hospital or health system must be determined before the outlay of capital. At a time when every health care dollar spent is being carefully considered, the question becomes: is this practice model a financial risk or is a TCC a viable way to reduce avoidable readmissions? A feasibility analysis can help to provide answers and guide project planning. This article describes how to conduct such a study as well as the financial assumptions that should be included when calculating the return on investment in a TCC.

\section{EVIDENCE BASIS FOR TRANSITIONAL CARE CLINICS}

With careful planning and collaboration, a TCC can grow hospital services, improve long-term patient outcomes, and improve the patient's experience of care. Exploring the literature on TCCs can provide the background information that will increase understanding of this relatively new care delivery model and improve the focus of the feasibility analysis. In addition, exploring evidence-based care delivery models and interventions will support developing best practices. 
Thus, when conducting a feasibility study, begin by performing a comprehensive, multilevel search of the current nursing, medical, and business literature on TCCs, transitional care delivery models, and theoretical frameworks applicable to the provision of transitional care services. Although the academic evidence remains limited on these topics, new studies continue to emerge as TCCs gain popularity. Search strategies should consider the variable nomenclature used to describe TCCs: these clinics have been referred to as "postdischarge clinics", "bridge clinics", and "aftercare clinics", and these search terms should be included to prevent limiting the discovery of information. A hand search of print and social media sites associated with targeted hospitals and health care systems may also prove helpful when searching for evidence on established clinics. Hospital and health systems often advertise TCCs on their websites and detail their services, hours of operation, and personnel.

Hospitalist, nursing, and geriatric medical practitioners have historically made the strongest contributions to the literature on transitional care. Many of these studies describe targeted interventions and care coordination strategies that can help those in the TCC planning stages develop their operational approach to service delivery. Programs such as Project Re-engineered Discharge (RED) and the Care Transitions Program have both consistently demonstrated readmission reductions and have been endorsed by institutions such as the Institute for Healthcare Improvement (IHI) and the Centers for Medicare and Medicaid Services (CMS). ${ }^{[2,7,9,10]}$ Despite those recommendations, however, these programs have not yet been widely utilized and many hospitals seem to prefer developing their own operational approaches to transitional care.

One care delivery framework that should not be overlooked when reviewing the evidence on transitional care is the Transitional Care Model (TCM). This model was created and tested by Dr. Mary Naylor and her colleagues at the University of Pennsylvania School of Nursing. With perhaps the strongest evidence for reducing readmissions, the TCM was developed to improve transitional care and reduce avoidable rehospitalizations of chronically ill older adults. It has been evaluated in over 20 randomized controlled trials with findings significant for decreased hospitalizations, lower overall costs of care, and increased patient satisfaction with care. Many TCCs are APRN-led and the TCM uses APRNs and an interprofessional care coordination model to improve long-term outcomes and promote continuity between care settings. ${ }^{[5]}$

\section{Components OF A FEASIBILITY STUDY}

After background information has been collected, formal evaluation of feasibility may begin. The goal of this eval- uation is to determine the market potential and financial viability of the project. ${ }^{[11]}$ The conclusions drawn from the analysis will help to determine the potential for success and provide a framework for the business plan. ${ }^{[12]}$

\subsection{Data collection: Define the target population}

The patient populations most at risk for hospital readmission have been well reported; however, each facility should review internal readmission data to verify that these patients are the ones most likely to utilize transitional care services. This drill down on institutional readmission data will also aid in decision-making about the clinic's business model and operational approach. The definitive scope of care provided in a clinic will likely vary by facility. A discussion of the mission, goals, and desired outcomes for the clinic can then follow. For example, one hospital may find it needs to focus its efforts on uninsured patients; other facilities may use TCCs as part of a comprehensive approach to chronic disease management. Reviewing 1 to 2 years of 30-day hospital readmission data will prove helpful in understanding the demographic characteristics of the readmitted patients and their health needs. The information obtained will also help to predict clinic volumes and will provide a general overview of the social determinants of health for the readmitted patient.

Admission and readmission diagnoses, length of time between discharge and readmission, discharge disposition, payor source, and descriptive information on patient ethnicity, age, and gender are all valuable data collection points. Once these foundational questions are answered and target patient populations are defined, the feasibility analysis can shift to the next phases of the study: determining organizational, market, and financial feasibility.

\subsection{Organizational feasibility}

Evaluating organizational feasibility helps project planners determine what administrative, management, and personnel resources will be needed to optimize success. For a hospitalbased clinic startup, several aspects of organizational feasibility should be considered:

- Organizational leaders' support for and engagement in clinic development.

- A strategy for identifying patients in need of clinic referrals.

- The complexity of care the clinic will offer.

- Available personnel with the qualities needed for dayto-day operations.

- The space, equipment, and technical support needed for operations.

- Credentialing, educational, or regulatory barriers to clinic operations, particularly for clinics utilizing 
APRNs.

- A strategy for documenting and communicating clinic encounters to ensure continuity with both hospital discharge and primary care providers.

Exploratory research methods will provide insight into many of these issues and help to define implementation hurdles. Interviews with organizational leaders, direct care providers, and allied support staff will help to identify the level of engagement for establishing a clinic and guide the development of an overall organizational plan. State boards of medicine and nursing are great resources for clarifying regulatory and collaborative practice issues, but the medical staff bylaws of each institution should also be reviewed for any barriers to practice.

The use of an evidence-based strategy to identify patients at risk for readmission and needing clinic referral should be considered. A protocol-driven approach such as the one used in the TCM can be useful in determining the resources that will be needed to deliver care and support day-to-day operations. The TCM uses evidence-based screening tools to identify patients at risk for readmission. Clinicians with advanced knowledge and skills are trained to deliver transitional care to high-risk patients within and across all health care settings. ${ }^{[5]}$ While this model is a good fit for the goals of TCCs, each facility must determine if the resources used in the model such as APRNs are readily available; in addition, the benefits of using this model must be balanced against the costs and time required for staff training and education. These factors could decrease momentum for the project or delay clinic implementation.

\section{MARKET FEASIBILITY}

A market analysis looks at current market trends and evaluates the industry and competition. There is substantial evidence that market conditions are favorable for the type of care delivery a TCC provides:

- The growing prevalence of chronic illness and hospitalizations suggests that the populations most at risk for readmission will continue to increase, which implies that a well-designed clinic could be highly utilized and have great sustainability.

- Compressed hospital care and increased patient and family caregiver responsibilities following hospital discharge have been shown to impact readmissions. In a 2012 survey sponsored by the American Association of Retired People Public Policy Institute and the United Hospital Fund, many patients reported using emergency services because they lacked educational support and access to resources for questions about health self-management or resources who could answer questions about care at home. ${ }^{[13]}$ The report indicated that care delivery venues outside the emergency room are needed; TCCs would be an ideal setting to meet this need.

- Even areas rich in health care providers and services need transitional care services. Assessments performed by two Houston Medical Center hospitals in 2013 concluded that despite the availability of community health and safety net services, the highest priority health needs for the community remain access to care; chronic disease management, including coordination of care and referrals; transitional care services; and other services that promote continuity of care.

Although the evidence is limited, the TCC market appears to be small but growing. Unfortunately, no database lists or registers TCCs. Thus, evaluating the local and national market must be done by using techniques such as hand searches. It is helpful to remember when conducting these searches that most TCCs are based in hospitals; exploring local markets might begin with a review of hospital websites. Also, remember that the nomenclature for TCCs is not standardized, so searches for established clinics should be done using alternative terms (see Section 2).

\section{Financial feasibility}

Determining financial feasibility gives financial stakeholders an indication of start-up and other capital requirements and projects the return on investment. One of the first steps in determining financially feasibility is deciding how a clinic should be designated or licensed. Licensing requirements are often determined by the setting and patient population using a clinic. Academic medical centers, student health centers, and clinics holding certain governmental designations may not require any licensing; some of these models will allow for enhanced funding and reimbursement. While hospital-based business models for TCCs appear to be the most common, other models such cost-savings (designed to meet pay-for-performance initiatives and mitigate penalties) and those with regulatory-based designation (i.e., FQHC) could also be designed to support transitional care. ${ }^{[14]}$ Hospital financial officers and billing and coding experts should be consulted to assist in navigating federal, state, and city regulatory and reimbursement options. The decision on which operational model is best will ultimately depend on institutional needs and the monetary resources available to that hospital or health care system. It may be helpful to create a reimbursement fee schedule specific to the clinic model proposed; this will aid in predicting revenue and in the creation of a cost-benefit analysis and business plan (see Figure 1). 


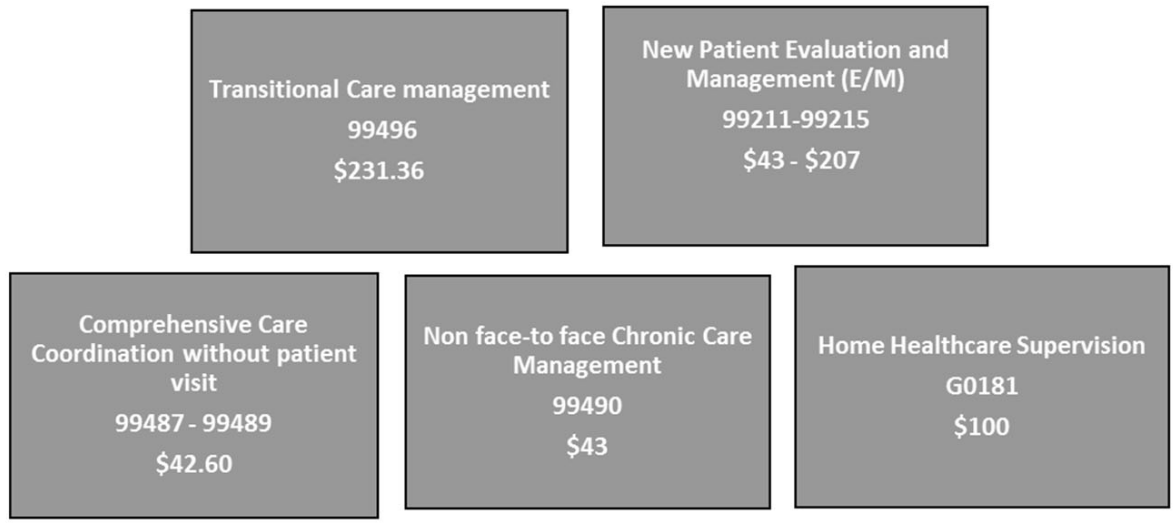

Figure 1. Additional reimbursement opportunities for TCC providers

Based on Centers for Medicare and Medicare (CMS) Healthcare Common Procedural Coding System (HCPCS), Current Procedural Terminology (CPT) codes can be used to predict revenue when considering the financial feasibility of a transitional care clinic

Cost considerations should reflect predicted construction, facility, supply, training and personnel needs. The benefit side of the equation will include reimbursement income based on the created fee schedule; however, a well-designed TCC has the potential to provide financial benefits and population health outcomes that are difficult to quantify: the mitigation of mandatory readmission penalties, decreased utilization of emergency services, even the business impact of improved Hospital Consumer Assessment of Healthcare Providers and Systems (HCPAPS) scores reflecting patient satisfaction with their discharge care. Improved patient safety around the discharge process, risk reduction related to discharge medication errors, enhanced care continuity, and the psychosocial support for families who now have access to care are all potential benefits that can be gained from establishing this innovative care setting.

The majority of hospital-based clinics utilize APRNs and pharmacists to deliver care in collaboration with a medical director. The staffing budget for this model might include the following: in year one, two APRNs budgeted at market salary would be used to run the clinic 7 days a week and provide extra coverage during the busiest days to improve efficiency. These APRNs could be new hires or could come from existing staff that has been repurposed. One APRN would operate as a clinic manger or director and provide general oversight of the clinic, staff, and day-to-day operations. Also included in the staffing budget would be funds for a medical director (at $20 \%$ of the medical director's time) who will spend time in outreach and education for the APRNs and primary care providers in the community. This salary would also help to cover additional liability for the physician and the time spent in the clinic assisting with clinical duties. Key personnel for this care delivery setting are pharmacists, who help with medication management and reconciliation and patient teaching, and social workers, who help with educating the participants on available community programs and working to improve community communication. A position may also be considered for an advanced practice paramedic who will be used in the community case management program to help reduce over-utilization of the emergency medical services and emergency room. Finally, a position should be included in the budget for an administrative assistant to help with data collection and reporting tasks.

The financial plan should include funds for costs such as fringe benefits like insurance, sick leave, and paid time off. Funds should be budgeted for travel, education telecommunications, office rental fees and supplies. The financial plan may even include money for written patient educational materials and assistance with medications.

Once expenditures and predicted revenue have been determined, a pro forma balance sheet can be created on the basis of the business model most suitable for the facility and the scope of clinic services that will be provided. When direct and indirect costs are being determined, consideration should also be given to the following:

- payor sources and fee schedules for both private insurance and Medicare patients,

- the fee schedule for self-pay patients,

- how no-show patients will impact operations and revenue,

- personnel needed for staffing and operations,

- inclusion of services such as transportation and parking, and

- potential strategic financial partners or space and support staff that can be shared.

A sample first year budget is provided in Table 1. 
Table 1. A simplified sample budget for a feasibility analysis of a transitional care clinic in start-up year

\begin{tabular}{ll}
\hline Budget Assumptions (Estimates) & Year 1 \\
\hline Expenses & $\$ 224,000$ \\
Rent (includes utilities) \$28 sq. ft. X 8,000sq ft & $\$ 20,000$ \\
Office refurbish (repurpose unused/existing space and assuming no new construction) & $\$ 0$ (repurpose existing staff) \\
Budget neutral personnel (NP, Pharm D, Social Services, clerical staff) & $\$ 0$ \\
Additional staffing costs (start-up year) & $\$ 215,000$ \\
Addition personnel (Medical Director @ 20\% of time; clerical; accounting; legal) & $\$ 75,000$ \\
Equipment/Supplies( paper products, furniture) & $\$ 25,000$ \\
Additional Direct Costs (marketing, promotion, phones, administrative fees) & $\$ 559,000$ \\
Total Expenses & $\$ 120,255$ \\
Revenue & $\$ 15,000$ \\
Based on TC Management Code 99496 10 patients/week @ 231.26 & $\$ 135,255$ \\
Technical Fees (based on lab and diagnostics bundle on 3 patients/week @ \$285.21) & \\
Total Revenue & \\
Approximately 34 patients/week to break even @ \$231.26/patient x 52 weeks (1,768 patients) & \\
and assuming technical fees for the same percentage of patients (33\%) & \\
Potential cost savings for reducing readmissions (Millions) & \\
\hline
\end{tabular}

While start-up costs can be substantial, they must be weighed against the substantial financial penalties that are being levied for avoidable readmissions. It is also important to note that while recent health care reform legislation established these penalties, new reimbursement codes for the provision of transitional care were also created; both physicians and APRNs may bill for these services. ${ }^{[3]}$ A TCC has great potential to provide cost-savings as well as a revenue-generating care delivery model for hospitals.

\section{Conclusion}

Supporting the health care needs of patients at risk for readmission is essential to maintaining the economic and functional viability of our health care system. The importance of reducing avoidable hospital readmissions will continue to increase over the next few years as CMS readmission penalties expand to more diagnoses. Continual improvement is necessary, not only to provide better outcomes after hospitalization but also to ensure that the aspects of care that connect health care providers are done with quality, cost, and continuity in mind. The model study described here demonstrates that TCCs may be an innovative and feasible approach to meeting this aim. It is time for all facilities to consider this strategy as way to reduce avoidable readmissions and improve health outcomes for their patients.

\section{CONFLiCTS OF INTEREST Disclosure}

The author declares that there is no conflict of interest.

\section{REFERENCES}

[1] Chronic diseases and health promotion. Centers for Disease Control and Prevention Web site. 2013. Available from: http://www.cdc. gov/chronicdisease/overview/index.htm

[2] Reduce avoidable readmissions. Institute for Healthcare Improvement Web site. 2014. Available from: http://www.ihi.org/ex plore/Readmissions/Pages/default.aspx

[3] Readmissions reduction program. Centers for Medicare and Medicaid Services Web site. 2013. Available from: http://www.cms . gov/Medicare/Medicare-Fee-for-Service-Payment/Acu teInpatientPPS/Readmissions-Reduction-Program.html

[4] Beresford L. Is a post-discharge clinic in your hospital's future. Hospitalist. 2011; 15(12): 1-42.

[5] Naylor M, Aiken L, Kurtzman E, et al. The importance of transitional care in achieving health reform. Health Affairs. 2011; 30(4): 746-754. PMid:21471497 http://dx.doi.org/10.1377/hlthaff . 2011. 0041

[6] Coleman EA, Smith JD, Frank JC, et al. Preparing patients and caregivers to participate in care delivered across settings: the care transitions intervention. J Am Geriatr Soc. 2004; 52(11): 18171825. PMid:15507057 http://dx.doi.org/10.1111/j.1532-5 $415.2004 .52504 . x$

[7] Boutwell A, Griffin F, Hwu S, et al. Effective Interventions to Reduce Rehospitalizations: A Survey of the Published Evidence. Cambridge, MA: Institute for Healthcare Improvement; 2009.

[8] Park HK, Branch LG, Bulat T, et al. Influence of a transitional care clinic on subsequent 30-day hospitalizations and emergency department visits in individuals discharged from a skilled nursing 
facility. J Am Geriatr Soc. 2013; 61(1): 137-142. PMid:23205951 http://dx.doi.org/10.1111/jgs.12051

[9] Barrett M, Baetzman S, Andrews R. US Agency for Healthcare Research and Quality. Overview of key readmission measures and methods. HCUP Methods Series Report \#2012-04. 2014. Available from: http://www.hcup-us.ahrq.gov/reports/metho ds/2012_04.pdf

[10] Stamp KD, Machado MA, Allen NA. Transitional care programs improve outcomes for heart failure patients: an integrative review. J Cardiovasc Nurs. 2013; 29(2): 140-54. PMid:23348223 http://dx.doi.org/10.1097/JCN.0b013e31827db560

[11] Zelman WN, McCue MJ, Glick ND. Financial Management of Healthcare Organizations (3rd ed.). San Francisco, CA: Josey-Bass; 2009.
[12] Brockman K. How to perform a feasibility study and market analysis to determine if an ancillary service makes sense. Orthop Clin North Am. 2008; 39(1): 5-9. PMid:18061763 http://dx.doi.org/10. $1016 / j$.ocl. 2007.09.004

[13] Reinhard SC, Levine C, Samis S. Home alone: family caregivers providing complex, chronic care. AARP Public Policy Institute. 2014. Available from: http://www.aarp.org/content/dam/a arp/research/public_policy_institute/health/home-a lone-family-caregivers-providing-complex-chronic-c are-rev-AARP-ppi-health.pdf

[14] Ota KS, Beutler DS, Loli AI. Postdischarge transitional care management: a reimbursable service in 2013. J Am Geriatr Soc. 2013; 61(4): 665-666. PMid:23581933 http://dx.doi.org/10.1111/jgs.1 2193 